\title{
Storytelling in Visual Analytics Tools for Business Intelligence
}

\author{
Micheline Elias $^{1,2}$, Marie-Aude Aufaure ${ }^{1,3}$, and Anastasia Bezerianos ${ }^{4}$ \\ ${ }^{1}$ MAS Laboratory, Ecole Centrale Paris, France \\ 2. SAP Research, Business Intelligence Practice, France \\ ${ }^{3}$ AxIS Research Group, INRIA Paris, France \\ ${ }^{4}$ Univ Paris-Sud \& CNRS, INRIA, France \\ michellelias@gmail.com, \\ Marie-Aude.Aufaure@ecp.fr, anab@lri.fr
}

\begin{abstract}
Stories help us communicate knowledge, share and interpret experiences. In this paper we discuss the use of storytelling in Business Intelligence (BI) analysis. We derive the actual practices in creating and sharing BI stories from in-depth interviews with expert BI analysts (both story "creators" and "readers"). These interviews revealed the need to extend current BI visual analysis applications to enable storytelling, as well as new requirements related to BI visual storytelling. Based on these requirements we designed and implemented a storytelling prototype tool that is integrated in an analysis tool used by our experts, and allows easy transition from analysis to story creation and sharing. We report experts' recommendations and reactions to the use of the prototype to create stories, as well as novices' reactions to reading these stories.
\end{abstract}

Keywords: Visual Storytelling, Business Intelligence.

\section{Introduction}

Stories are one of humanity's communication structures and storytelling a means of passing on wisdom and culture. Individually and collectively, stories help us make sense of our past and reason about the future. Johnson 17] and Maclntyre [21] argue that story narrative also goes beyond communication, it is also a process of extracting meaning from events, that is central to human experience and conduct.

It is thus not surprising that intelligence analysts, who make sense of data, identify links between disparate pieces of intelligence, and communicate their findings to decision makers, are interested in storytelling. Their analysis process is supported by the construction of stories and narratives, both during sense-making and during presentation of results. Bier et al. [3] point out that a story is a powerful abstraction to conceptualize threats and understand patterns as part of the analytical process, and story structures and storytelling is the means to present the analysis results. As analysts continue to work with increasingly large data sets, data visualization has become an incredibly important asset both during sensemaking analysis, and when communicating findings to other analysts, decision makers or to a broader public [15, 27]. 
Given the importance of storytelling in different steps of the analysis process it is clear there is a need to enhance visual analysis tools with storytelling support. Nevertheless this process is not simple $[33,20]$, as analysts need to work within very large data resources and highlight and explain items or events of importance and their connections to their audiences. Despite the growing use and research work on storytelling and narrative visualization in the visualization domain $[26,9,15]$, related research on the domain of BI has not equally progressed. Our work attempts to rectify this.

In Business Intelligence (BI) analysis, the most popular visualization tools are dashboards. Dashboards [8] are collections of visual components (such as charts or tables) on a single view [12], that permit analysts to explore their data and quickly view different aspects of complex datasets. Nevertheless, simple collections of visual representations cannot be interpreted by untrained audiences; to become meaningful they require interpretation and explanation, often presented in a story narrative.

We attempt to answer the following research questions: What are the actual practices of BI experts in creating and communicating visual stories to their audiences, and do current BI visualization tools support well this story creation and storytelling process? How can we enhance BI visual analysis tools with narrative capabilities, and are these capabilities effective in communicating analysis stories to others?

Our work makes the following contributions:

(1) Interviews with expert BI analysts (story "creators" and "readers"), provide a better understanding of current practices in creating BI stories. BI stories are an asynchronous, visual, and interactive means of transmitting and sharing information on data between analysts and their audience. They include visualized data (dashboards, charts or tables) and detailed explanations on the story structure in the form of presentation(s), detailed textual annotations and external resources such as wikis. (2) Current tools fail to support the storytelling process, which remains cumbersome and requires frequent switching between software (analysis tools, screen captures, text editors, presentation tools, etc.). We emphasize the need for storytelling support, and extract requirements for enhancing BI tools with visual storytelling capabilities. (3) Following these requirements and a user-centered design approach, we implement a storytelling prototype incorporated in an existing visual analysis dashboard, to fluidly transition from analysis to story creation and reading. (4) We report on feedback from BI experts on the usefulness of the prototype as a communication and teaching medium, and from BI novices reading a story on its effectiveness for story communication.

\section{Related Work}

Stories are series of ordered events and facts, and their connections (Oxford English Dictionary). In intelligence analysis, it is furthermore an abstraction used by analysts to conceptualize threats and understand patterns in the analytical process [3], and communicate their findings to others [2].

Stories in Business. In recent years, organizations and their leaders have identified the importance and value of narrative and anecdotal information conveyed in the form of stories [29], to present either complex ideas, the context and details of crucial information, or personal interpretation of that information [28]. 
Research conducted to date has demonstrated the value of storytelling to improve organizational structure [24] and collaboration quality [23, 6], socialization and adaptation of new employees [7, 18, 19], organizational and financial success $[4,5]$, innovation and new product development [22], and teaching and learning [16].

The majority of this work is a meta-analysis of the effect of storytelling within an organization, rather than identifying the storytellers' needs in terms of supporting and enhancing the storytelling process as is our case. Moreover, the stories themselves discussed in this work, relate to the transmission of information and knowledge within an organization, mostly in textual or verbal form, rather than in visual form. The widespread use of visualization dashboards in the domain is a more recent development [8], and so is the transmission of knowledge between organizations (dedicated BI analysis organizations and their clients). Thus storytelling needs in the domain have evolved. In Section 3 we explain current practices in visual knowledge transmission and we take a step at characterizing current problems and needs more precisely.

Stories in Sense Making. Baber et al.[2] point out that contemporary theories of sense making rely on the idea of 'schema', of a structure to organize and represent factual information, as well as the knowledge, beliefs and expectations of the people who are engaged in sense making. They can thus be considered as a collection of narratives. They further discuss the formalism of stories in sense making, and how the most effective stories are organized around the actors in the stories, their actions and rationale, events, their context, and most importantly the relationships between these. As argued by Bier et al.[3], for effective collaboration and communication we need to use less text, and organize knowledge around entities (people, places, things, times etc.) rather than free form text. Similarly, Pirolli \& Russell [25] propose the mapping of intelligence facts and insights into frames, that can be expressed in a variety of forms including stories, maps, organizational diagrams or scripts. It is thus clear that conducting intelligence analysis, communicating findings, and organizing knowledge in stories, has a strong visual component that represents entities and their connections.

Stories in data visualization. Often text or audio transmit the main story, while visualizations support the story or provide details. Comics and flowcharts are special narratives relaying mostly on visuals rather than text. Recently, we have seen an increase in integrating complex visualizations into narratives in many news organizations [11], journalism reports (e.g. New York Times, Washington Post, the Guardian), and television reports (e.g. Gapminder ${ }^{1}$ ). Segel et al.[26] explore different aspects of narratives from a variety of sources and identify distinct genres of narrative visualization.

In the business intelligence domain the main visualization tools are dashboards [8], collections of multiple visual components (e.g. charts, tables) on a single view [12]. BI dashboards permit users to interpret data at a glance, and are very popular (e.g. [Dundas ${ }^{2}$, Oracle bi $10 \mathrm{~g}^{3}$, Xcelsius, Spotfire ${ }^{4}$, Tableau ${ }^{5}$ ). But as Wojtkowski and

\footnotetext{
${ }^{1}$ http: //www.gapminder.org, 2010

2 http: //www. dundas.com/microsite/dashboard

${ }^{3}$ http: / / www. oracle.com/technetwork/middleware/

bi-enterprise-edition/

${ }^{4}$ http: //spotfire.tibco.com/

${ }^{5}$ http: / /www.tableausoftware.com/
} 
Wojtkowski [33] point out, dashboards and other visualization tools used to analyze complex data cannot simply tell stories. They need to be "tailored" to accommodate storytelling by better highlighting items of importance within very large data resources [14], in a way that is efficient for the storyteller and clear for the audience.

Some visualization systems began to integrate storytelling [20]. GeoTime [9], a geo-temporal event visualization system, integrates a story system that shows events in space and time, hypertext linked visualizations, and visual annotations to create an environment for both analytic exploration, and story creation and communication. The design of the tool was based on real user needs and has been evaluated with intelligence analysts. Wohlfart and Hauser [32] present a system for demonstrating to audiences the path followed in analyzing 3D volumetric data, while providing audience with limited interaction with the 3D data. Both these systems deal with a single visualization/chart seen over time or from different views. Thus their designs are not necessarily applicable to the multi-dimensional and multi-chart BI visualizations where the connections and links between data are not as clear.

Storytelling tools in BI are not yet as advanced. Systems like Sense.us [15] and Tableau ${ }^{5}$ allow analysts to visualize data, conduct analysis, and store a history of the exploration, that can serve as a step towards creating a story. Many Eyes [31], Tableau Public ${ }^{5}$ and Sense.us [15] publish interactive visualizations online, and permit collaborative analysis through comments on a single visualization, creating an evolving analysis. This collaborative annotation can be seen as a step towards a collaborative knowledge narrative, where an analysis story could be extracted from the visualizations and comments. Nevertheless, these tools do not provide explicit means to indicate story progression and highlight relationships between multiple visualizations (seen in dashboards), that are key to intelligence analysis and communication [2].

\section{Interviews}

To support visual storytelling in BI, we first investigate current practices in transmitting BI analysis results, and identify challenges experts face currently when creating and sharing their stories, or reading and interpreting stories of others. We interviewed $5 \mathrm{BI}$ experts in a leading business intelligence development company, with experience from 6 months to 12 years. Participants' job descriptions included development project manager, project manager, development director, data warehouse engineer and delivery manager. Three experts used dashboards and communicated their analysis or read analysis from others daily, while two several times a week. Interviews were held in person or by phone and lasted over 1 hour (Table 1). We report next our major findings. Note that they hold true for all BI reporting tools available today, as indicated by our experts' experience with multiple tools, and our own investigation.

Table 1. Experts', experience, dashboard use and interview duration

\begin{tabular}{l|ccccc}
\hline Experts & P1 & P2 & P3 & P4 & P5 \\
Experience & 7 years & 6 months & 6 years & 3 years & 3 years \\
Dashboard use & daily & weekly & weekly & daily & daily \\
Duration & $90 \mathrm{~min}$ & $120 \mathrm{~min}$ & $60 \mathrm{~min}$ & $60 \mathrm{~min}$ & $60 \mathrm{~min}$ \\
\hline
\end{tabular}




\subsection{Current Practices: BI Reports}

All experts communicate their analysis or read analysis from others in the form of BI reports. These contain an entire dashboard, often accompanied by several single charts and tables. Their purpose is to help monitor aspects of business performance, by highlighting with charts key performance indicators (KPIs) that indicate success of project management and progression of development teams. Our experts were report creators, but occasionally acted as readers of others' reports.

A BI report can be a single page, with only a title and a dashboard (Fig. 1.). This one-page report summarizes the most important data, and can serve as a starting point for longer reports, up to 50 pages in length, that give more details. Details can be additional visualizations, tables, annotations, links to the data used in the visualizations, and finally block text, although all participants preferred limiting text to 5\% of the space in a report. When possible, creators want to make their reports "live", with interactive visualizations for the audience to explore. Thus BI reports have a very strong visual component, with little text added for explanations. The one page report is preferred by clients like company managers, that want an "at-a-glance view" of a project status, and that see many such reports during a day. Longer detailed reports are used to communicate findings to other analysts, project teams and product owners.
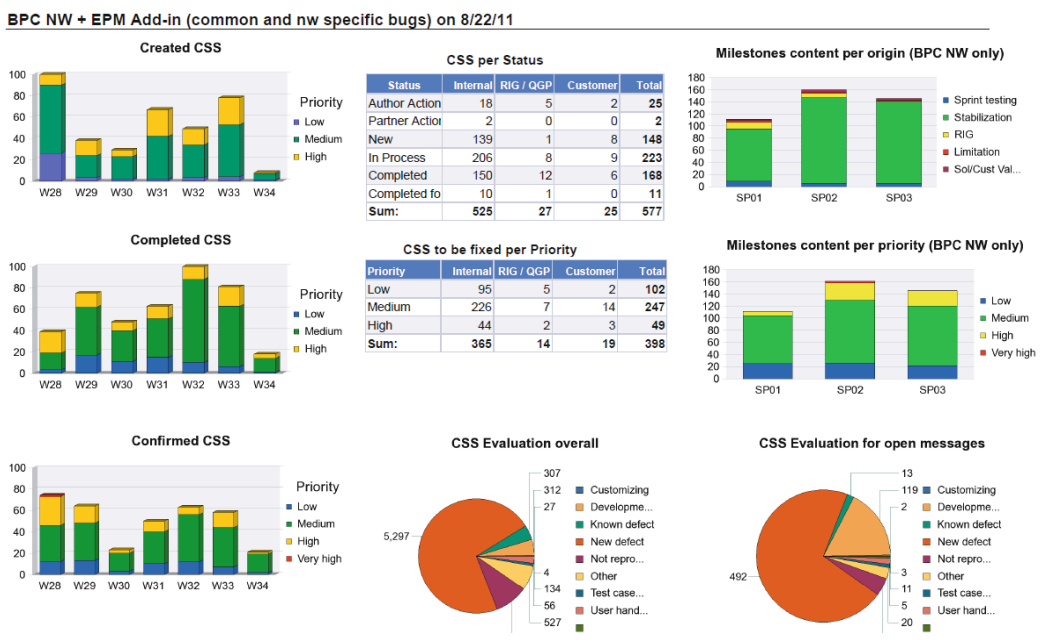

Fig. 1. One page BI report from an expert: dashboard presenting bug status in a project. Note the lack of annotations/explanations, aside from chart titles and data dimensions.

Experts mentioned that BI reports are used to (i) answer specific questions (e.g. What is the code development progress in project $X$ the last 3 weeks?); (ii) investigate specific data (e.g. Investigate the increase of bugs in section $\mathrm{Y}$ of project $\mathrm{X}$ ); (iii) manage conflicts and highlight problems (e.g. Team X completed less use cases than planned, because test team Y did not complete testing); and (iv) interpret past data and predict future trends (e.g. Given last year's sales, what is this year's projection?).

BI reports are nevertheless only one of the tools used to communicate analysis findings, and are not a complete BI story. 


\subsection{Current Practices: Supporting Material for BI Storytelling}

Experts explained that reports are difficult to understand without detailed explanations from the creator. Thus they don't represent a complete BI story on their own. Before publishing a new report, the creator provides an introduction session to report readers. During this (usually one hour long) session, she explains with a presentation the entire story and goal of the report, the meaning of each chart, the relations between different KPIs in different charts, as well as in what sequence to read it. Thus their verbal explanations and presentation slides are ways for creators to explain their analysis path. These presentations often show relational diagrams, text explanations and interpretations, highlight specific visualizations or parts of visualizations using colored highlights, arrows and other symbols. The report creator often draws by hand on a chart the ideal data values, to help compare the current situation to target goals.

The audience can ask questions during or after the training sessions, through emails or arranged meetings. Content similar to the presentation, is often put on a wiki page to answer follow-up questions. The session recording, wiki page, and presentation slides, are made available to the audience as reminders and reference material. Experts explained that this material is not a complete story either, as it does not include the visualization and data details shown on the report.

Thus a complete BI story is a collection of visual representations of the most important data followed by further data details (BI report), accompanied by instructions on how to read the visualizations (order, connections, importance) in the form of presentations and verbal or textual instructions on a wiki. Although creators present a desired way to view data, this structure is not enforced: the audience can pursue the story in a different sequence and dig for data details in the report. Thus a BI story differs from a simple fixed sequence presentation that prohibits exploration. Its goal is to communicate analysis findings and supporting evidence.

\subsection{Current Practices: Teaching BI Storytelling}

According to our experts, all the material making up a BI story is also a tool for training analysts. As a recently trained creator explained (P2), this material taught her the key aspects of BI reports and how to interpret them. When analysts start out they read in detail older reports and their supporting material to understand how to analyze visualizations, see relations, and identify important points and their link to KPIs.

Experts mentioned that designing a new report is hard and requires a lot of experience, thus they often use a template that they modify according to their needs. Three $(\mathrm{P} 2, \mathrm{P} 3, \mathrm{P} 4)$ stressed the influence of a senior creator $(\mathrm{P} 1)$, and their reliance on her BI report templates to create their own. They still occasionally contact the senior creator when facing difficulties or are unsure of the clarity of their message in a report.

The supporting material is also often reused. All experts explained that data (and thus reports) change, but often the structure of BI stories repeats itself. Moreover, they sometimes create a sequence of reports on dynamically evolving data (e.g. sales over several years). These stories change very little. Thus material on how to read reports can be re-used by adapting it to new stories, nevertheless, experts pointed out it can be a tedious and repetitive process. 


\subsection{Current Practices: Collaboration in BI Stories}

Experts explained that report creation follows several iterations of communication with a client (usually a decision maker like a manager or executive), who is one of many possible report readers. Thus the focus and structure of the story changes and evolves through the communication between the story teller and the story reader over time, and stories become the collaboration product of story tellers and readers. This process does not appear in most other visual analysis domains and visualization storytelling, where the reader's needs do not directly factor into the creation of the story.

This interactive report evolution process can be heavyweight (through phone calls, emails, etc.) and take several iterations to iron out, while the communication details are often lost between different versions of the report. At each iteration, creators explore the desired data using interactive visualization dashboard tools, create initial visualizations, provide additional information and details about the analytical techniques used, and finalize reading paths (i.e. how to read a report).

Once the story is finalized, it serves to clarify connections in the data and answer specific questions analyzed by BI analysts (see section on BI Reports). These answers often result in more detailed or tangent questions from the audiences, and the need for analysts to conduct side data investigations and generate new stories. As in other visualization domains, BI stories are thus a visual communication medium between storytellers and their audiences, but contrary to other domains, there is an open communication loop, where audiences can continue to ask for new stories.

\subsection{BI Storytelling Challenges}

All experts use an in-house reporting tool, that gives access to different data sources and can extract interactive dashboards, charts and tables from their dashboard analysis system. It also provides the capability of adding text. Three (P1,P3,P5) also used Excel for creating less complex reports. Our experts identified several issues with this process, which are similar to other BI analysis and reporting tools.

When extracting interactive visualizations from their analysis tools, metadata and annotations from their analysis are not extracted and have to be recreated. Moreover, the annotation capabilities of the report creating software are very limited. They cannot annotate specific data points, while sequences and connections cannot be displayed graphically but have to be explained in one of the supporting material. Finally the report creation tool can be in-house software. Thus to share reports, creators often extract static snapshots of the tool output and save it in PDF format for their readers.

Some report creation tools give access to interactive visualizations and dashboards through hyperlinks. Our experts like this option and add these links to their noninteractive reports (PDF, Excel) and presentations. Readers within the organization may have access to the in-house reporting tool, and can then interact with individual visualizations or dashboards (e.g. query and filter data, or drill down/up). This interactivity is lost when reports are communicated outside the company.

To overcome the shortcomings of reporting tools, creators are forced to provide the supplementary material (presentations, wiki pages) and when possible links to interactive visualizations for in-company clients. They described this process as limiting and requiring a lot of work duplication. 
We requested clarification on two points regarding the possible integration of analysis and storytelling: (i) Given that analysis dashboards are exploratory environments that allow users to interact fully with data, should such interactions be allowed in the storytelling? (ii) If creators had the opportunity to create their entire story with all support material in one place, how would they prefer the story to be visualized?

Interactivity. Due to the constraints of current reporting tools, shared reports are often non-interactive when accessed outside the organization. When creators were asked if they want the visualizations in the reports to be completely interactive and encourage readers to interact with them (e.g. using drill down/up, filter, link \& brush), four (P1,P2,P3,P5) of our experts prefer to have interactive visualizations that permit linking and brushing (i.e. data selection). But they would limit the more advanced interactions such as drill down/up or filtering. They felt that all the data needed to tell the story should be displayed clearly in the report without the need to explore the data further. The fifth (P4) would not be opposed to fully interactive visualizations. Thus authors feel business stories should be mostly author-driven and constraint, known to work best when the goal is storytelling or efficient communication [26].

Story Templates. Going from the current practices of storytelling (BI report, presentations, wiki) to a dedicated storytelling tool is not straightforward. We thus showed our experts a group of story templates identified by Segel et al. [26] to see if they met their needs. All chose the "Annotated chart" as the preferred template (Fig. 2), with the modification that it should have multiple charts on the same page (dashboard) that they can annotate. Four experts (P1,P2,P3,P5) identified "Partitioned poster" as a potential template, where the side list of charts display details that support the main chart in the central region. Three (P1,P2,P5) mentioned that the "Slide Show" template is useful both as a means to focus on attention on each chart and zoom to details, and as a step-by-step presentation that clarifies the analysis path and the ideal reading sequence. One expert (P5) found the "Flow Chart" useful for showing some business scenarios, like following bugs during development (discovered, tested, fixed etc.). Another (P3) found the "Comic strip" useful template, but with added annotations.

So besides the templates identified by Segel et al.[26], for BI stories we need a new template that consists of an annotated dashboard. Our users attempt to do this with their current reporting tools, but they are limited (cannot annotate detailed points and relations), or they create it manually in their presentations.
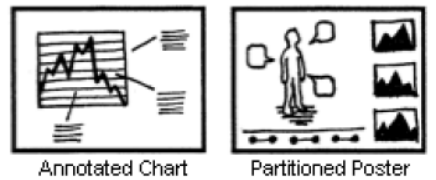

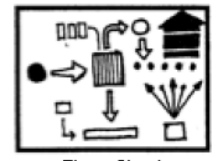

Flow Chart

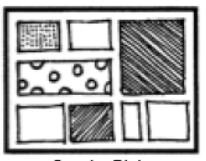

Comic Strip

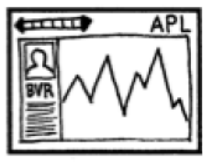

Slide Show

Fig. 2. Genres of visual narratives templates from Segel et al. selected by our BI experts 


\section{$4 \quad$ Participatory Design Session}

We brought back the senior BI expert that trained other analysts (P1), to identify the best design for a BI storytelling tool. She provided us beforehand with one of her old reports and supplementary material. During this session we gave the expert a copy of her material, and several cut-outs of the entities in the report (charts, titles or tables). She was also given "narrative aids", such as arrows, lines, numbered items, grouping containers of different colors, and annotation bubbles. We asked her to construct a stand-alone story of the report on an A3 paper, with accompanying audio if necessary, in a way that it can be interpreted by a general audience with no training. The session lasted 2 hours and was recorded.

Fig. 3 shows the final version of the manually created story by the expert, where we can see the intense usage of annotations and explanations, connected by arrows and lines. Other structures used by the participant were chart grouping rectangles (enclosures for placing charts that need to be read together), sequence markers (what needs to be read first), and highlight markers (to draw attention to part of the story).

We asked our expert how she would prefer the readers to see the story, as a static image or an animated presentation. She explained that both are needed, the static representation shows the "entire story and gives context", whereas the animation "focuses the audience where I want". She then played out for us how she wanted the animation to be presented, explaining when and how to zoom to specific areas of the story. This play-back was recorded using static shots.

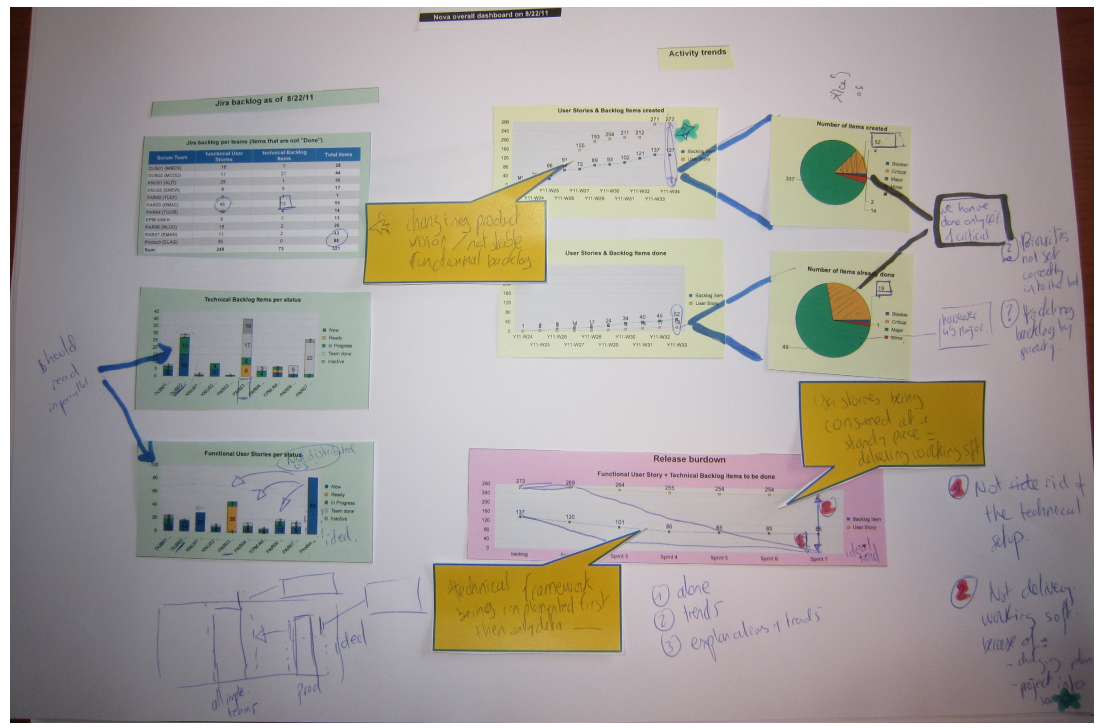

Fig. 3. Story created by an expert during the design session 


\section{$5 \quad$ Requirements for Creating BI Stories}

Based on expert self-reporting, BI reports, the main communication medium of BI analysis, consist of snapshots of visualizations and textual descriptions, made in a different environment than the one used for analysis. These reports are fact-based and can be interpreted only by an expert audience. To increase their audience, report creators use supplementary material, like wiki pages, presentations, and when possible links to interactive visualizations. Based on our experts' interviews and a participatory design session, we identified a set of requirements for enhancing BI analysis tools with storytelling capabilities:

R1. Fluid Transition. Analysis tools used to explore data and create visualizations are different from report creation tools. Exporting visualizations from the first to the second to create a BI story costs time and effort, and limits the possibility of embedding meta data or annotations created during the analysis. To ensure that creators do not recreate information, they require a fluid and integrated way to transition from their analysis and meta data associated with it, to report creation.

R2. Integration. To tell their stories, BI creators need tools that combine all materials used currently in their story creation: BI reports, interactive visualizations, ways to indicate story structure, highlighting capabilities, presentation of the story in sequence, and textual or audio explanations.

R3. Narrative visual aids. Report creators need to add focus expressions to draw attention to specific visualization data, such as highlighting, coloring, annotating and zooming. They also require ways to indicate reading sequence (e.g. vectorial references, like arrows [13][30]). These are not available in reporting or BI analysis tools.

R4. Interactive visualizations. Visualizations on shared reports are often noninteractive when read outside the organization. A storytelling tool should have completely interactive visualizations, although the way that readers interact with the data should be limited (by default to brushing and linking) and be controlled by the creator. This balance has been identified as a challenging aspect of storytelling [20].

R5. Appropriate BI Story templates. BI stories have specific structure not necessarily shared by other story narratives identified by Segel et al.[26]. Our experts identified templates of interest (Fig. 2) and highlighted the need for a new template that consists of an annotated dashboard.

R6. Reuse. Although BI reports and data changes from analysis to analysis, often the underlying structure of BI stories remains the same. It is thus important to be able to easily reuse the structure of stories created within the tool both for stories of evolving data and similar future stories.

R7. Optional playback. Readers should be provided with a static representation of the entire story to get an overview and explore it on their own pace, as well as a guided playback to help them follow analysis paths that not easy to discover and understand the important points according to the creator.

Such storytelling support would facilitate the creation and learning to read BI stories. While the scientific visualization storytelling tool 32] supports R3,R4,R7, and GeoTime [9] supports R1 and R3, we are not aware of any BI dashboard or other multi-chart visualization system that provides all the above functionalities. 


\section{BI Narrative Prototype}

Following these requirements, we extended an existing dashboard exploration tool to support the creation of BI stories.

\subsection{Exploration/Annotation Dashboard (Fig. 4)}

The user starts from a traditional analysis dashboard, a collection of coordinated (synchronized) charts connecting one or more data sets. It provides advanced exploration capabilities such as data selection and filtering, and OLAP functionality (e.g. drillup/down) [1]. We build our narrative tool on top of an existing visualization dashboard system [10] that supports annotations on "data targets", such as charts or tables (e.g. a bar-chart) or parts of them (e.g. a specific bar in the chart). Annotated data targets are highlighted in the dashboard and an icon indicates the number of attached annotations. A list of all annotations is also available on the right.

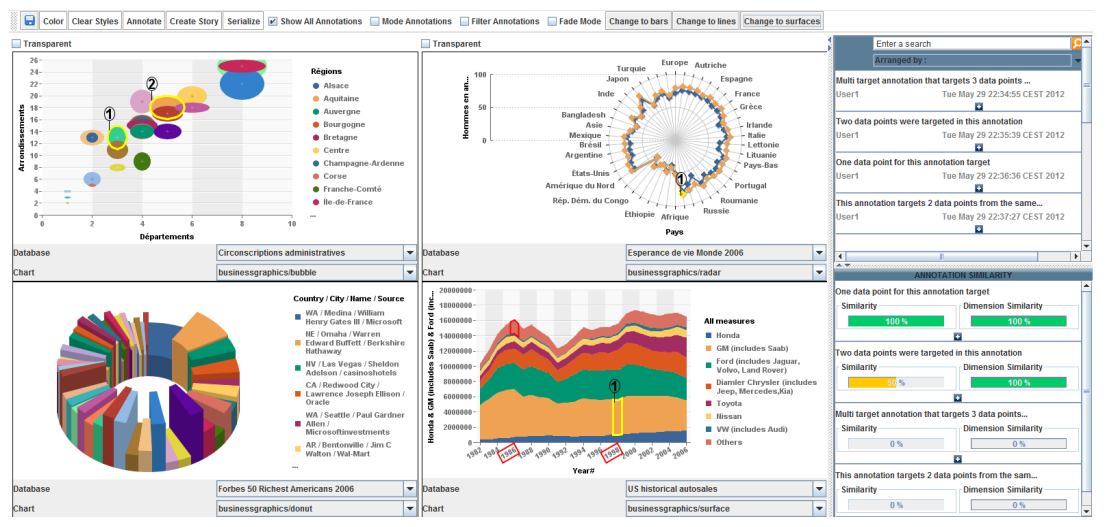

Fig. 4. Dashboard with visualizations created during analysis on the left, and list of annotations added to different data contexts while exploring analysis visualizations on the right.

After or while conducting her analysis on the dashboard, the analyst can create a story. The menu option "Create story", opens up the narrative board window. All visualizations and their annotations (annotation text + data targets highlighted) are placed in the narrative board. Thus the analyst can transition fluidly (R1) from analysis to story creation. Because dashboards can present evolving data, but a story can be an instance in time, by default each visualization is placed on the narrative board with a time-stamp to indicate when the visualization was taken from the data.

\subsection{Narrative board (Fig. 5)}

Here users create a BI story. Visualizations and annotations from the dashboard appear on the main window, arranged by default as in the dashboard (to support the dashboard template identified in our interviews R5). Users can resize and relocate all 
story entities freely, or choose to organize them using other appropriate narrative templates (R5). They can edit visualization titles, annotations of entities, and add new text entities. Users can add visual entities that indicate relations, grouping and emphasis $(\mathbf{R 3})$ to help create stories, available at a tool palette on the right. The story can be seen in: a static representation, where all entities remain one screen; and a playback representation, where entities are highlighted sequentially according to an authordefined sequence (R7). We categorize the entities available on the narrative board as:

1. Information entities: visualizations, text, annotations. These are mainly imported visualizations and annotations created during the analysis. For annotated visualizations, the annotation text, the visualization, as well as the annotated data target (specific part of the visualization that is annotated), are all defined as story entries. Users can also place text anywhere on the narrative board to further explain their story.

2. Relational entities: arrows, lines, html links. A story can include relationships between entities, such as causality. We provide visual arrows, lines, html links and other vectorial references to define relations between entities (Fig. 5.c).

3. Organization entities: visual grouping and sequences. Previous work [25] and our interviews emphasized the need of visual grouping of entities that are to be seen together in a story. We support this with entity grouping borders. Our experts also indicated the need to define a reading sequence for each entity, to help readers move through the story. We achieve this by allowing users to define the order of appearance of all entities through a sequence list (Fig. 5.c). Users can change the entity's sequence in the list, delete, or rename any entity. They can also define a playback time for each entity, a time for the entity to be in focus in the playback presentation mode. Audio can also be recorded for any entity, to be played during the story playback.

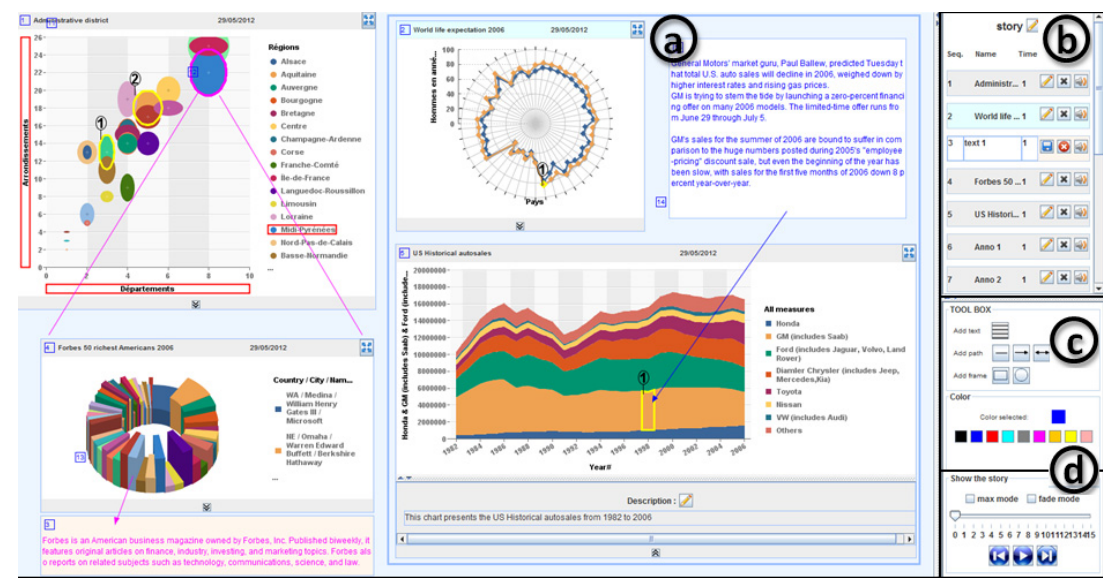

Fig. 5. (a) Narrative board containing all story entities arranged by the story teller: information, relational (arrows), organization (groupings) and emphasis entities, and numbers indicating author reading sequence. This sequence appears in a list (b), where authors can define playback properties and add audio commentary. Below is a pallet of relational and organization entities (c), and the Playback panel (d) to control playback through the story time line. 
4. Emphasis entities: highlighting and zooming. To focus reader's attention to a specific story entity, users can add color highlights to any entity (e.g. a visualization, an arrow, or text entity), or select and highlight parts of visualizations (e.g. a bar in a bar chart visualization). This color highlighting can be present in the static story presentation (always visible), or during playback (the color highlight appears at a specific point in the story timeline). Besides color highlighting, users can add zooming highlighting to any story entity, to take place in a specific point in time during playback.

Playback. As discussed in (R7) our experts desire 2 ways of showing their stories to their readers, a static overview version that provides context and allows free exploration, and a playback that shows a recording of the author suggested path. Our narrative board acts as the static version. We provide three options of animated playback, giving focus to entities in sequence for a given duration. In the "color highlight", entities in focus change color to grab attention (default playback). In the "max playback", entities in focus are zoomed-in to the maximum possible size, taking up the entire narrative board. Finally, in the "fade mode", during playback, all entities except the entities in focus fade out (Fig. 6). As mentioned, authors can record audio to accompany the playback. Readers can pause the playback at any point to explore the story.

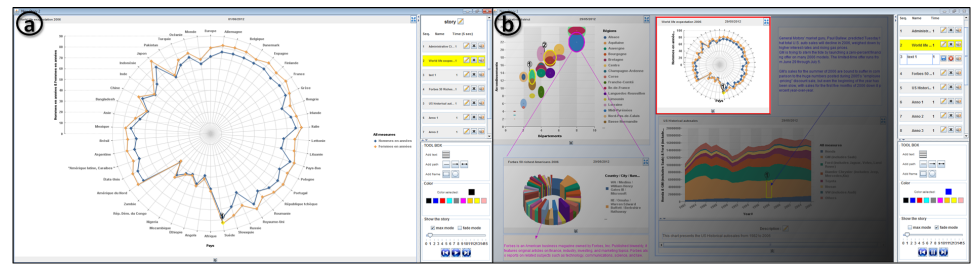

Fig. 6. Two playback modes: Max (a), and Fade (b)

Interactive Visualizations and Exploration. Our visualizations are imported with time-stamps referring to a "snapshot" of the dashboard data at the time of the story creation. Nevertheless, they are still connected to that version of the data and are still live. Thus users can still interact with them to perform brushing and linking actions (R4) and explore them further. By default we deactivate advanced interactions like drill-down/up, but authors can reactivate them through the visualization's properties.

The creators may also decide to take a new snapshot of the data (i.e. change the timestamp for the visualizations, and so the values of the data). Thus they can reuse the story structure for a new version of data (R6), which is particularly important for evolving dynamic data. Finally, users can select and replace any visualization with another, even from another dashboard loaded in the system. Thus they can reuse the story structure not only for dynamic data, but also for completely different datasets.

Thus our system integrates all the material used currently in BI story creation (R2): BI reports (visualizations and annotations), interactive visualizations, ways to indicate story structure, highlighting, optional story presentation in sequence, and text or audio explanations. It can then be shared with readers with access to the prototype. With respect to other BI systems, ours is one based on a user-centered design based and expert user requirements, and is unique in supporting annotations that link multiple points in the story, different story playbacks, and providing templates for BI stories. 


\section{$7 \quad$ User Feedback}

We conducted two user feedback sessions to assess the usability and effectiveness of the system, both from the creator's and the reader's perspective.

\subsection{Story Creation by Experts}

We invited back two of the interviewed experts (P1,P2), and showed them the system in individual one hour sessions. Expert P1 was the experienced analyst (7 years) that has trained others. Expert P2 has been an analyst for 6 months and was trained by P1. They explored the prototype, saw story examples, and created stories themselves.

General Feedback and Recommendations. Both experts were very enthusiastic with the prospect of having access to such a system for their work. They found it easy to use and helpful, especially as it is integrated in the exploration/annotation dashboard tool. As P1 mentioned it "saves me from recreating any charts or annotations to present to others". It also saves time and effort not just in report creation, but also in communicating reports: as P1 explained, by using the prototype, there is no need for an explanation presentation to clients, for wiki pages or recreating interactive charts.

Both creators reaffirmed that story reading must be guided by the story creator, else the goal of the story may be lost. Currently they enforce this by the order of explanations used in their BI reports. They commented that the prototype supports this well with the numbered sequence for entities, while the playback (with fade out and zoom) guides readers through the story and keeps their focus on one entity at a time. But they appreciated that they can create and show a story on one page (even with scrolling), as opposed to the current multi-page reports, because as P1 mentioned "people tend to read only the first page, and explore less the following pages". And because it gives readers flexibility "the reader can always understand the position and link between parts and the overall story" and "look for more details when she wants".

Participants liked the ability to use the annotated dashboard template, saying that "this is how we want to present our analysis story, similarly to our dashboard". They both commented that annotations attached to data points are very important in pointing out to readers important data values "I add a lot of manual arrows to point to annotations that refer to specific data points on a chart, and their ideal value".

Both experts suggested our system should support two types of BI narrative stories: (i)Fixed stories, that present snapshots of datasets at specific points in time, yet are interactive (e.g. for filtering), to be shared as tutorials, explanations, or reports. This is the default case in our system. And (ii) Online stories, that present dynamically evolving data, and can have the same analytic scenario regardless of data values. Thus stories may have the same chart descriptions (e.g. what data is shown), the same KPI relations, and the same reading sequence. Here visualizations in stories are no longer snapshots, but are updated with data changes. We have implemented this extension.

Collaboration and Communication with BI Stories. Expert P2 commented on how this storytelling prototype can also be used as a means to evolve stories. Multiple analysts can integrate their own comments and knowledge in the story, encouraging peer learning, but also collaboratively creating more complete and detailed stories. 
Expert P1 mentioned that she could envision using the system to iterate the definition of the story directly with her clients (a process conducted now by email or phonecalls). She envisioned clients adding themselves on the story further explanations on specific entities and their relations (to explain patterns), possible summaries of decisions they took based on the report, or highlighting what information is missing. This goes beyond collaborative analysis and storytelling: it directly empowers readers and becomes a medium to communicate what they want from the story.

In both cases, the prototype moves from a one-way communication to a collaboration medium, where the authoring of a story opens-up and evolves with the contributions of many users, and acts as an archive of knowledge and different points of view. Such a system, our experts explained, needs to clearly differentiate between the contributions of individual authors. We are currently exploring this extension.

Finally the story can be archived and used by new analysts that learn how to create stories (comment from our trainer analyst). P1 stressed how important such an archiving is for knowledge passing between analysts. The recently trained P2 stated the tool can help him further improve his reports by looking at the story structure of others.

\subsection{Story Reading by Novices}

We then ran a second session to evaluate the prototype from the reader's perspective, and thus close the story communication cycle. We conducted 40 to 50 minute sessions, with 5 BI novices. All were IT professionals, knew what a dashboard is but had never worked with one. Two had heard of BI reports but never used one. Participants were asked to (1) Read a BI report created by one of our experts, (2) Read a BI story (created by an expert in the previous session), and (3) Explore our prototype.

The story presented the progress of a development project from different perspectives: General (how is the project evolving in terms of finished code in a sprints time line, how many code components are added to a waiting list, and how many critical, major or minor code components are done each week); and Detailed (the progress of each development team in coding and testing each code component). The development is not progressing according to plan because many bugs fixed are not critical, whereas new bug reports coming in add critical bugs to the waiting list. The bottom right chart in the report highlighted the problem and the rest provide details.

Reading the Report. When given the report, all readers read the first page dashboard from left to right and up to down (which is not the "author" suggested order). They understood the goal (progress of a project) and what the charts displayed (e.g. bugs in waiting list) with the aid of chart titles and legends. But they all struggled to find the problem illustrated in the report. Only one participant noticed that the project development is not progressing over time, but she could not understand why. This supports our experts' comment that reports cannot be read without the supporting material.

Reading the Story. Participants found that reading a story was easier "it showed the facts in an understandable manner". All 5 readers found the system easy to use, understood the story, and were able to answer correctly comprehension question related to the story content. We report here our main observations and readers' comments: 
Memorization: All users remembered even detailed aspects of the story and only went back to the system when answering detailed quantitative questions (e.g. to retrieve numbers from charts), while all general comprehension questions were answered without going back to the story visualizations and comments. They tended to answer using the same terms used by the expert analyst in the story. When asked similar questions on the report, they went back to it each time to search for answers.

Confidence: Readers were not confident in their understanding of the report as they had to draw their own conclusions. They expressed worry that they may have misunderstood or not noticed important points. While when reading the story, all felt confident, as their interpretations are confirmed by the analyst's comments.

Guidance: All readers appreciated the guidance in reading the story, both static and playback. They commented on the importance of both modes: "the static mode permits to understand the whole story" and "dig for facts", while in playback it "is easier to follow" the story sequence and focus on important data. Four participants preferred the fade playback, and three the max mode when focusing on an entity.

Understanding: All users understood the story using our tool, and found it easy to read and interpret. They commented on how it was easy to find answers both to qualitative and quantitative questions. The story structure showed clearly what is the problem, how to analyze it and how to find the cause. While they described the report as ambiguous, as they couldn't identify the relation between charts or KPIs.

Transmitting knowledge: All readers found annotations very helpful in explaining the relationships between charts and KPIs, and in teaching them the analysis logic they should follow. Four mentioned that the system can "aid in transmitting different knowledge in the company between different users", and two would like to use the system to communicate with their team their own data (even if they are not analysts).

Engagement: Three readers got very engaged with the story, as "stories are more encouraging than static reports". They began searching in charts to find how this problem can be solved, exploring the story outside the suggested structure.

The comparison of BI reports without supplementary material to our story prototype is clearly unfair and our goal is not to prove its superiority in terms of communication value. It serves to illustrate how our stand-alone prototype could be an effective means of communicating BI stories without additional material. A comparison of our tool against the collective BI story material used today is future work, but our experts already identified its superiority in terms of saving time on report creation, the sharing of interactive stories, and being a medium for collaborative story evolution.

\section{Discussion and Conclusions}

We identify for the first time the current practices and needs of BI storytelling. BI analysts often organize visually and communicate their analysis story to others. Nevertheless their tools do not allow easy transition from visual analysis to storytelling. They often use multiple tools, replicate work, and train their audience to understand their analysis. This reinforces the need for explicit storytelling support, missing in most existing visual analytics systems even in other domains, and provides insights on how to address this need for dashboards and other coordinated view systems. 
Based on interviews and a paper prototyping session with expert BI analysts, we derived requirements for extending BI visual analysis dashboards to support storytelling. These can be adopted as-is by designers of BI systems, or inspire and inform visual storytelling research in other domains. For example providing an author suggested story structure, seeing the story in either a playback or it in a static overview, and imposing exploration constraints to interactive visualizations, can apply to other domains. Others, like the need to reuse story structure, may be BI specific and need to be reexamined for other domains. Using these requirements we implemented an extension to a BI dashboard, allowing transition from analysis to storytelling.

We evaluated our prototype with story creators and found the requirements and prototype meets their needs. More importantly, they highlighted the potential of storytelling tools as (possibly asynchronous) two-way communication channels, were authors communicate their findings, and readers also pose questions directly on the story. This empowers readers, informs authors of limitations of their story, and captures the evolution of the story. In BI it also accelerates story creation, that is highly client driven, and whose details are often lost in emails and phone calls. Tools can also act as collaborative platforms, where multiple authors refine, create variations of, and archive stories. Our findings open questions regarding the archiving and navigation mechanisms of story versions, trust in authoring changes, and maintaining a clear story focus. These questions open up exciting new research avenues.

We then presented a story from an expert to novice readers, to test the understandability of stories in our system, and its potential as a stand-alone tool for communicating BI stories. Readers understood the stories without training, and answered complex comprehension questions. Few previous works (e.g. [9]) evaluate visual storytelling systems from the reader's perspective, a crucial step in the communication cycle.

Our system was identified by experts as having great potential for reaching a broader audience, as little to no training is required to read stories, and for training analysis. Although designed as a stand-alone tool, it does not aim to replace expert analysts. As an expert mentioned, ours and other storytelling tools can aid novice analysts or readers to quickly read analysis results and focus their questions to experts on more complex aspects, such as methodology, goals, content details, etc.

We are finalizing our prototype to give to BI analysts. We aim to verify requirements gathered through expert self-reported with more direct observations of the use of the tool and its impact on current analysis and communication practices.

\section{References}

1. OLAP and OLAP server definitions. The OLAP Council (1995)

2. Baber, C., Andrews, D., Duffy, T., McMaster, R.: Sensemaking as narrative: Visualization for collaboration. In: VAW 2011, U. London College (2011)

3. Bier, E.A., Card, S.K., Bodnar, J.W.: Entity-based collaboration tools for intelligence analysis. In: IEEE VAST, pp. 99-106 (2008)

4. Boje, D.M.: The storytelling organization: A study of story performance in an officesupply firm. Administrative Science Quarterly, 106-126 (1991)

5. Boje, D.M.: Stories of the storytelling organization: A postmodern analysis of disney as "tamara-land". Academy of Management Journal, 997-1035 (1995) 
6. Boyce, M.E.: Collective centring and collective sense-making in the stories and storytelling of one organization. Organization Studies, 107-137 (1995)

7. Brown, M.H.: That reminds me of a story: Speech action on organizational socialization. University of Texas at Austin (1982)

8. Cutroni, J.: Google analytics. OReilly Media, Inc. (2007)

9. Eccles, R., Kapler, T., Harper, R., Wright, W.: Stories in geotime. In: IEEE VAST, pp. 1926 (2007)

10. Elias, M., Bezerianos, A.: Annotating bi visualization dashboards: Needs \& challenges. In: ACM CHI 2012, pp. 1641-1650 (2012)

11. Farhi, P.: CNN hits the wall for the election (2008), http: / / bit. ly/cnn-wall

12. Few, S.: Show me the numbers: Designing tables \& graphs to enlighten. Analytics Press

13. Forceville, C.: Educating the eye? kress and van leeuwens reading images: The grammar of visual design (1996). Language and Literature 8(2), 163-178 (1999)

14. Gershon, N., Page, W.: What storytelling can do for information visualization. Commun. ACM 44(8), 31-37 (2001)

15. Heer, J., Viégas, F.B., Wattenberg, M.: Voyagers and voyeurs: Supporting asynchronous collaborative visualization. Commun. ACM 52, 87-97 (2009)

16. Helmer, J.E.: Between horses: An ethnographic study of communication and organizational culture at a harness track. U. of Illinois at Urbana-Champaign (1989)

17. Johnson, M.: The moral imagination. University of Chicago Press, Chicago (1993)

18. Louis, M.R.: Surprise and sense making: What newcomers experience in entering unfamiliar organizational setting. Administrative Science Quarterly (1980)

19. Louis, M.R.: Organizations as culture-bearing milieu. Organizational symbolism: Monographs in organizational and industrial relations, pp. 39-54 (1983)

20. Ma, K.-L., Liao, I., Frazier, J., Hauser, H., Kostis, H.-N.: Scientific storytelling using visualization. IEEE Computer Graphics and Applications 32(1), 12-19 (2012)

21. Maclntyre, A.: After virtue. University of Notre Dame Press, Notre Dame (1981)

22. McWhinney, W.: A health confluence (1995) (unpublished paper)

23. McWhinney, W., Battista, J.: How remythologizing can revitalize organizations. Organizational Dynamics, 46-58 (1988)

24. Mitroff, I., Kilmann, R.H.: Stories managers tell: A new tool for organizational problemsolving. Management Review (1975)

25. Pirolli, P., Russell, D.M.: Introduction to this special issue on sensemaking. In: HumanComputer Interaction (2011)

26. Segel, E., Heer, J.: Narrative visualization: Telling stories with data. In: IEEE InfoVis (2010)

27. Shrinivasan, Y.B., van Wijk, J.J.: Supporting the analytical reasoning process in information visualization. In: ACM CHI 2008, pp. 1237-1246 (2008)

28. Snowden, D.: The art and science of story or 'are you sitting uncomfortably?' Business Information Review, 215-226 (2000)

29. Sole, D., Wilson, D.G.: Storytelling in organizations: The power and traps of using stories to share knowledge in organizations. Harvard Graduate School of Education (2002)

30. Tversky, B., Heiser, J., Lozano, S., MacKenzie, R., Morrison, J.: Enriching Animations, volume Learning with animation. Cambridge University Press (2007)

31. Viegas, F.B., Wattenberg, M., van Ham, F., Kriss, J., McKeon, M.: Manyeyes: a site for visualization at internet scale. IEEE TVCG 13, 1121-1128 (2007)

32. Wohlfart, M., Hauser, H.: Story telling for presentation in volume visualization. In: IEEE EuroVis 2007, pp. 91-81 (2007)

33. Wojtkowski, W., Wojtkowski, W.G.: Storytelling: its role in information visualization. In: European Systems Science Congress (2002) 